\title{
La démission de la Commission européenne : scandale et tournant institutionnel (octobre 1998 . mars 1999)
}

Didier Georgakakis

\section{(2) OpenEdition \\ Journals}

Édition électronique

URL : http://journals.openedition.org/conflits/814

DOI : $10.4000 /$ conflits.814

ISSN : $1777-5345$

Éditeur :

CCLS - Centre d'études sur les conflits lilberté et sécurité, L'Harmattan

Édition imprimée

Date de publication : 1 juin 2000

ISBN : 2-7384-9946-5

ISSN : $1157-996 X$

Référence électronique

Didier Georgakakis, « La démission de la Commission européenne : scandale et tournant institutionnel (octobre 1998 - mars 1999) », Cultures \& Conflits [En ligne], 38-39 | été-automne 2000, mis en ligne le 20 mars 2006, consulté le 30 mars 2021. URL : http://journals.openedition.org/conflits/814 ; DOI : https://doi.org/10.4000/conflits.814

Ce document a été généré automatiquement le 30 mars 2021.

Creative Commons License 


\title{
La démission de la Commission européenne : scandale et tournant institutionnel (octobre 1998 - mars 1999)
}

\author{
Didier Georgakakis
}

Pour en restituer très brièvement le développement chronologique, les scandales à la Commission européenne ont pour origine un ensemble de malversations dénoncées dans la presse belge en août 1998. À la suite de ces révélations, le scandale a peu à peu pris forme dans la presse puis au Parlement. Ce dernier dépose une motion de censure finalement rejetée mi-janvier, mais, à cette date, est désigné un comité des sages pour éclairer les affaires. Rendu le 15 mars, son rapport met en cause Edith Cresson pour faute personnelle et le Collège pour «mismanagement ». Dans la nuit, la Commission démissionne.

La restitution sous la forme apparemment la plus objective de cette chronologie de «ces six mois de grand déballage " comme le titrait Libération vient sans conteste conforter l'interprétation la plus courante de ces événements ${ }^{1}$. La chaîne continue entre le fait, le contrôle et la sanction renforce comme naturellement l'idée d'une «victoire de la démocratie ». Cette interprétation s'est imposée avec d'autant plus de facilité qu'elle a été répercutée par l'ensemble des protagonistes de l'affaire: par les journalistes avec l'argument de l'émergence d'un "espace public européen ${ }^{2}$, par les parlementaires qui invitent à y lire une victoire du contrôle opéré par leur institution, et même par le Collège des Commissaires. À un moment - il faut le rappeler - où Jacques Santer comptait bien se présenter à sa propre succession, il pouvait escompter quelques bénéfices d'une démission collective réinterprétée comme une opération "mains propres" délibérément souhaitée. Au printemps 1999, qui voit se dérouler conjointement des campagnes pour les élections européennes et un ensemble d'élections nationales importantes, cette interprétation pouvait également s'appuyer 
sur la dénonciation des manières opaques de l'«eurocratie » qui est devenue une constante du discours politique en Europe ${ }^{3}$.

Cette thèse de la victoire de la démocratie a sa part de vérité, au sens où il s'est bien joué ici quelque chose qui est de l'ordre de la modification d'une configuration institutionnelle, mais elle peut être prise dans, au moins, deux autres sens : comme un " argument" constitutif de la dynamique du scandale et utilisé par des acteurs très différents pour faire advenir le réaménagement institutionnel qu'ils appellent de leurs vœux ; et comme un signifiant suffisamment fort et, simultanément, labile pour rendre lisible un univers structurellement complexe et conjoncturellement marqué par un très haut degré d'incertitude. En invitant à déplacer le regard de la "thèse démocratique " vers ses usages, ces deux derniers sens permettent de mieux comprendre ce qui s'est joué. Plus qu'à une victoire de la démocratie, la dynamique de ce scandale renvoie en effet à des stratégies relevant de situations très hétérogènes et animées par des acteurs dotés de motifs assez souvent opposés, mais qui ont trouvé dans les affaires une opportunité de peser sur les transformations institutionnelles en jeu dans l'agenda 2000 et la réforme interne de la Commission. C'est là que porte l'essentiel des transformations qui se sont jouées dans ce scandale: non pas dans d'irrémédiables acquis, mais dans un ensemble de jeux-test qui ont donné corps au scandale et avec lui à de nouvelles façons de lire et de pratiquer les institutions européennes.

Aussi est-ce sur ces « réaménagements » institutionnels que voudrait porter cet article, en s'articulant moins sur le développement chronologique du scandalestricto sensu que sur ses versants interne et externe. Revenir sur la dynamique du scandale dans une perspective empruntant à la sociologie des crises politiques invite en effet à montrer que le scandale dont la Commission européenne a fait les frais est né de la rencontre entre deux types de transformations. D'une part, des transformations liées à un ensemble de tensions internes à la Commission, et plus particulièrement aux effets du déplacement nordiste et "managérial» de son centre de gravité sur le climat administratif; d'autre part, une redéfinition des relations entre les membres du Collège, les parlementaires et les journalistes passant par la mise en cohérence de chacun de ces groupes et le recours à des savoir-faire politiques nouveaux au sein des institutions européennes. En ce sens, et au-delà d'un cas de scandale, c'est sur les différentes facettes de la politisation des institutions européennes que voudrait revenir cette contribution ${ }^{4}$.

\section{«Politique » interne et gestions de l'affaire}

Pour qu'existe une affaire et que celle-ci dérive en scandale, il faut qu'un fait se trouve qualifié ou étiqueté comme hors normes, que cette qualification soit partagée et qu'il s'engendre une mobilisation ${ }^{5}$. C'est dire, comme le dit après tout si bien l'expression "étouffé dans l'œuf ", que c'est dans un va et vient entre l'interne et l'externe que se nourrit la dynamique d'un scandale. Aussi la question des raisons pour lesquelles ont été "sorties » ces affaires invite-t-elle à faire porter l'éclairage sur un ensemble de " frustrations ${ }^{6}$ internes. Ces frustrations ne tiennent pas ici à la redistribution de ressources matérielles, mais plutôt à la rencontre entre le pouvoir accru des commissaires et les pressions que les réformes de l'organisation de la Commission font peser sur les fonctionnaires. Sous l'effet de ces transformations, s'est en effet opéré une rupture des liens de solidarité entre le Collège des commissaires et les fonctionnaires 
qui n'a pas été pour rien dans la définition du climat dans lequel les « affaires » ont été révélées puis qualifiées de «scandale».

\section{De la gestion confidentielle des affaires à la définition d'un nouveau climat}

Le climat dans lequel les affaires ont été sorties puis transformées en scandale a sans doute des origines externes. Contre une vision trop «administrativiste», les fonctionnaires européens gagnent à être analysés pour ce qu'ils sont aussi : des agents sociaux situés dans une ville très particulière. Or Bruxelles représente le lieu central d'une crise de la représentation qui s'est imposée de façon particulièrement puissante en Belgique. Il faudrait plus que quelques lignes pour en inventorier les effets. Au-delà d'une humeur qui porte à la valorisation de la pureté, cette crise passe par la modification des attentes et des grilles de lecture de la presse belge (celle précisément d'où ont émergé les affaires) ; elle passe aussi par l'attestation de l'efficacité du recours à des répertoires de mobilisation dont les «marches blanches» sont des points culminants. Les fonctionnaires européens ne sont pas étrangers à ce contexte, qu'ils le signalent en évoquant la "déprime ambiante ", ou en répercutant les blagues belges relatives à l'affaire Dutroux et la crise du régime. Mais si ce contexte de la crise de la représentation belge a compté, c'est surtout qu'il est venu donner du sens au climat trouble qui s'est imposé au sein de la Commission européenne. C'est dans les transformations liées à l'émergence de ce « climat » qu'il faut chercher les raisons pour lesquelles la Commission s'est trouvée dans l'impossibilité de maintenir à l'intérieur la gestion des « affaires ».

L'origine de l'affaire est, à sa manière, politique, puisque les faits qui ont conduit au scandale de la Commission sont liés soit à des pratiques clientélistes, soit à l'externalisation du traitement d'un ensemble de dossiers qui a longtemps tenu lieu de remède au décalage croissant entre l'extension du volume d'activité de la Commission et la relative stagnation de son budget de fonctionnement. Mais ces procédés étaient auparavant considérés comme des cas-limites et dont il fallait bien s'accommoder. Avant le scandale, la représentation des fraudes relève en effet essentiellement de dispositifs techniques et secrets au sein de la Commission. La nature technique de ce traitement est garantie par l'existence d'un corps de spécialistes, l'UCLAF, l'unité de coordination de la lutte anti-fraude, qui comprend cent cinquante agents environ, dont la plupart sont détachés des administrations nationales (douaniers, magistrats) et qui est, au départ, surtout conçue comme un organisme de contrôle des subsides communautaires dans et par les Etats membres ${ }^{7}$.

L'existence de cette unité rappelle le poids des agents spécialisés dans le traitement des affaires mais aussi que tous les entrepreneurs de morale, selon l'expression d'Howard Becker ${ }^{8}$, ne sont pas des agents extérieurs : c'est du reste - on y reviendra - lorsque les premiers sont déstabilisés au profit des seconds et qu'ils ne tiennent plus les définitions de la fraude et de la sanction légitime que s'opère la "dérive » vers le scandale. De ce point de vue, ce n'est pas un hasard si l'on connaît peu de chose des " précédents » aux affaires de l'an dernier. Précisément, ce ne sont pas devenus des affaires. D'une part, l'UCLAF tenait le monopole de la qualification de la fraude et d'autre part, la circulation de l'information s'opérait dans des liens assez directs avec le Collège des commissaires. La chronologie de cette affaire illustre bien la confidentialité relative du travail de 
l'UCLAF et sa capacité à maintenir à l'intérieur les jugements possibles sur la gestion de la Commission. L'UCLAF a bel et bien enquêté sur les faits et sanctionné le hautfonctionnaire jugé responsable, mais le silence a longtemps dominé. C'est plus tard qu'ont été publiées des enquêtes sur les précédentes « affaires » européennes, comme dans Le Monde diplomatique d'octobre 1998 puis dans l'ouvrage de François d'Aubert sorti aux lendemains de la démission'.

Dans cet état du jeu, l'« économie » des relations qui caractérise le traitement de la fraude est servie par le devoir de réserve. Indépendamment d'une obligation statutaire, ce dernier est particulièrement fort à la Commission. Si l'accès à l'information concernant les programmes est particulièrement libre, la possibilité d'une réflexivité sur l'organisation de la Commission est très largement encadrée, comme l'ont remarqué Marc Abélès et Irène Bellier ${ }^{10}$ au point qu'elle s'observe tout particulièrement dans les relations entre les fonctionnaires et un "enquêteur extérieur ». Cette difficile réflexivité ne tient pas seulement à ce que l'institution tient les ressources (notamment en termes de sanction ou d'avancement). C'est aussi que pour ces membres d'une institution longtemps marginale et menacée, il relève de la défense commune vis-à-vis de l'extérieur, par ailleurs entretenue par tout un travail de stylisation des eurocrates, de valorisation de l'histoire des pères fondateurs et d'une éthique de la virtuosité très proches de celle des groupes hiérocratiques ${ }^{11}$.

Des transformations importantes sont intervenues sous les Commissions Delors. L'autorité "charismatique » prêtée à Jacques Delors ne tient pas seulement à sa personne mais à des changements dans l'organisation de la Commission et notamment dans sa politisation, comme en témoignent le poids croissant des cabinets, la consultation directe par les commissaires d'un ensemble d'intérêts, etc ${ }^{12}$. Cette "politisation" de l'administration européenne n'a pas disparu avec la Commission Santer. Les propriétés politiques des Commissaires en témoignent ${ }^{13}$. Mais avec la Commission Santer sont intervenues d'autres modifications dont l'effet apparaît déterminant sur la naissance du «climat " très particulier dans lequel a pris corps le scandale $^{14}$. Pour beaucoup, l'arrivée de Jacques Santer a surtout signifié un rééquilibrage de la Commission sur le versant nordique et managérial. Cette dimension a tout particulièrement été incarnée par deux commissaires provenant des pays nordiques entrés en 1995 dans l'Union européenne et qualifiés d'« anges gardiens » du nouveau Commissaire. Le premier, Erkki Likkanen, commissaire socialiste finlandais et surtout ministre de l'économie qui a préparé l'entrée de la Finlande a en charge le budget et le personnel et il est à l'origine de plusieurs projets de réforme de l'organisation interne de la Commission européenne ${ }^{15}$. En obtenant le poste de Commissaire « responsable du contrôle financier et de la lutte anti-fraude » - c'est son titre officiel - Anita Gradin, socialiste suédoise, a pour sa part reçu la mission de rompre avec les pratiques «sudistes" de la Commission Delors et de remettre de l'ordre, notamment en accentuant la pression de l'UCLAF.

Lors de leur déclaration d'investiture, l'intention de " changer de régime ", c'est-à-dire d'accélérer un ensemble de réformes internes est manifeste. Elle s'incarne par la suite dans des tentatives de réforme de structure. En ce qui concerne les réformes initiées par E. Likkanen, plusieurs projets ont été formulés, incluant notamment la réforme du statut, le recours officialisé et accru à des sociétés extérieures ou encore la possibilité de licencier les fonctionnaires « underperforming ». Ces projets de réforme ont été mis en scène sous une forme volontairement "moderniste" (grand-messe médiatique, recours à des consultants, etc.) et ont été interprétés comme une remise en cause du 
dialogue social interne à la Commission et du statut des fonctionnaires, tous deux très importants symboliquement. Mais surtout - et c'est là qu'intervient le climat - ces projets de réforme ont donné lieu à une mobilisation sans précédent. La journée d'action d'avril 1998 a été particulièrement suivie et elle a plus encore représenté un point d'orgue dans le processus de désolidarisation entre le Collège et l'administration de la Commission. Pour la première fois, des directeurs généraux, postes réputés politiques et proches du Collège, ont manifesté contre ce dernier. Comme le montre le courrier des lecteurs de la revue interne de la Commission, la journée d'action a été en outre suivie d'un ensemble de «débats internes" mettant aux prises les «modernistes » et partisans du «nord» avec les «anciens " partisans du «sud» de l'Europe. En bref, ont affleuré toute une série de clivages en nette rupture avec l'ordinaire des luttes entre Directions générales ${ }^{16}$.

Mais là n'est pas la seule transformation apparue avec la nouvelle Commission. La réforme interne impulsée par le nouveau Collège s'incarne également dans la définition d'une politique de moralisation des pratiques du métier de fonctionnaire. Les deux aspects sont étroitement liés puisque la réforme du statut porte aussi sur les fraudes. Le 13 novembre 1997, a ainsi lieu une réunion d'information d'E. Likkanen sur les « insuffisances professionnelles, les irrégularités financières et les fraudes » et ces projets sont, dès cette époque, dénoncés de façon virulente par les organisations syndicales « pour l'amalgame entre insuffisance professionnelle et fraudes relevant de la criminalité » et le "simulacre de dialogue social conduisant à la réforme du statut » (tract Union Syndicale, 18 novembre 1997). Mais cette politique de moralisation est plus directement portée par Anita Gradin, la Commissaire en charge de la lutte antifraude. Rencontrant les débats sur les nécessités d'une transparence accrue des affaires européennes initiés par la Finlande et la Suède lors de la conférence intergouvernementale, cette politique passe par un ensemble de mesures symboliques comme le projet de revenir sur des privilèges réputés, tel le droit au colis d'alcool et le tabac hors-taxe souvent évoqués lors de la grève contre le projet de réforme du statut. Elle s'incarne également dans le renforcement de l'UCLAF, la volonté d'initier plus d'enquêtes en interne et une requalification de la fraude, passant par la disqualification de la "gestion Delors» ou d'un ensemble de pratiques "sudistes» au profit d'une définition plus «nordique » de la rigueur - dont les mesures sur l'alcool ne sont qu'un exemple. Si elles n'ont pas fait grand bruit à l'extérieur - à l'exception d'un article dans European Voice - les sanctions en 1996 à l'encontre d'un fonctionnaire grec et d'un français ont semble-t-il pris valeur d'exemple et, au sein de la Commission, elles ont contribué à l'alimenter l'idée que les fonctionnaires étaient désormais placés sous haute surveillance.

\section{L'extériorisation des tensions}

Le renforcement de la politique anti-fraude a sans conteste permis que toute une série de "problèmes » se transforment en " affaires ", pour reprendre la typologie de Pierre Lascoumes ${ }^{17}$. Mais cette transformation s'est opérée à un moment bien particulier. En intervenant dans le contexte des conflits liés à la réforme interne de la Commission, la dénonciation des fraudes par l'UCLAF et le Collège a contribué à accroître le sentiment de dépossession des fonctionnaires dans le même temps que les luttes internes avaient ouvert la voie à un ensemble de ripostes auparavant impensables. Le scandale est né de ce décalage entre cette politique de moralisation et le climat conflictuel interne à 
l'administration. Le départ de l'affaire, l'existence des «fuites» qui ont permis l'enquête des journalistes puis la diffusion du rapport confidentiel de Paul van Buitenen au groupe « Vert » du Parlement permettent de s'en convaincre.

Les effets de ce décalage des situations se donnent tout particulièrement à voir dans le départ de l'affaire. La première révélation de la Lanterne tient en effet à une action en justice au Luxembourg. Or cette dernière ne vient pas de nulle part. Elle tient à une enquête interne - leur nombre s'est de fait multiplié - et qui conduit au limogeage d'un haut fonctionnaire, français, responsable de la gestion du programme Echo. Dans ce contexte, l'affaire est l'occasion d'un premier engrenage. Le fonctionnaire refuse sa résiliation en des termes qui sont très proches de ce «refus de payer pour les fautes politiques de commissaires" que montrent les tracts des organisations syndicales. Aussi il attaque en justice la société responsable des malversations et réputée forte de soutiens politiques à la Commission (Perry-Luxe). Or non seulement la société riposte en se retournant contre la Commission mais son patron, Claude Perry, se livre à un travail de mobilisation contre la Commission. C'est dans le cadre de cette riposte qu'il est conduit à rencontrer Jean Nicolas, le journaliste de La Lanterne qui a révélé publiquement l'affaire.

Dans l'ouvrage qu'il a consacré à l'Affaire ${ }^{18}$, le journaliste Jean Nicolas montre bien la collaboration de Claude Perry, et du reste celle, conjointe, du fonctionnaire d'Echo. La rupture des transactions ordinaires qui les liaient et leur mise sur la touche les conduisent à se retourner contre la Commission et à « donner » les dossiers accumulés. Il reste que d'autres fuites vont permettre la constitution du dossier et surtout la mobilisation de l'ensemble de la presse. S'il est pratiquement impossible de mettre des noms sur les auteurs de ces fuites, celles-ci n'apparaissent néanmoins pas sans liens avec les nouveaux clivages internes à la Commission, et notamment ceux qui divisent Collège et fonctionnaires. Sans aller toujours jusqu'à la dénonciation, cette "rupture » entre Collège et fonctionnaires s'accompagne en effet d'une modification de la frontière du dicible et de l'indicible pour ne pas dire une disposition assez largement partagée par les fonctionnaires à "vendre la mèche ». Cette modification s'observe dans le cadre des conversations et dans les entretiens avec des fonctionnaires européens : la liberté de parole est beaucoup plus grande durant la période et, du reste, elle s'observe a contrario dans les silences d'après la démission. Elle se donne également à lire dans la nature virulente des tracts syndicaux, les menaces de sanction contre l'exécutif politique de la Commission et surtout celles, à peine masquées, de faire des révélations à la presse qui apparaissent notamment à l'été 1998. Pour mémoire, la journée d'action d'avril ne s'est pas déroulée en vase clos mais la veille d'un Conseil des ministres à Bruxelles relatif à l'agenda 2000 : c'est dire qu'elle était explicitement tournée vers les médias et les acteurs du Conseil et non pas en direction des responsables de la gestion du personnel.

En même temps que se déplace cette frontière du dicible et de l'indicible, c'est la frontière entre l'intérieur et l'extérieur de l'institution qui est en jeu. Tout un ensemble de coups se jouant auparavant par tracts et messages internes interposés tendent à "sortir» de ces relations de face à face pour se jouer par presse interposée. L'annulation du concours de recrutement des fonctionnaires A8 de septembre 1998 à la suite de «fuites » portant sur le contenu des sujets en est un bon exemple. Il est difficile de dire si les sujets ont été sortis pour nuire au Commissaire Likkanen, mais ce qui est sûr, c'est qu'en tant que responsable du personnel et donc des opérations de concours 
et de recrutement, ce dernier s'est trouvé en première ligne. Les syndicats ne se sont en effet pas privés de le mettre sur la sellette au motif du gaspillage occasionné par le concours et de son "incompétence» - c'est la riposte à son projet de licencier les personnels «underperforming" - et cette interprétation du gaspillage et des problèmes de management à la Commission a très largement couru dans la presse. $\mathrm{A}$ l'inverse, le Commissaire n'a pas hésité à évoquer la thèse d'un sabotage par le personnel de la Commission destiné à le déstabiliser dans le quotidien belge Le Soir, argument à son tour vivement dénoncé par le Syndicat TAO-AFI (tract du 14 octobre 1998).

Cette «humeur révélatrice » permet de mieux comprendre que le scandale se nourrit d'un ensemble de coups dont l'enjeu tient dans le rapport de force entre les syndicats et le Collège. La révélation de nouvelles affaires ou la diffusion de nouvelles informations à l'extérieur est du reste entretenue par la concurrence entre les différents syndicats de fonctionnaires. Dans un numéro du "Renard déchaîné » - le titre est déjà explicite - le syndicat Renouveau et Démocratie, qui se situe sur la gauche de l'Union Syndicale, donne à voir la mesure des révélations qu'il entend faire, en dénonçant les privilèges et les avantages dont disposent les représentants syndicaux majoritaires. Au regard des usages stratégiques des affaires, il n'est, au total, pas vraiment surprenant qu'aux accusations contre les commissaires «du Sud" (Edith Cresson, Emma Bonino et Manuel Marin) s'ajoutent bientôt les révélations sur les commissaires les plus engagés dans la réforme de la Commission, notamment Anita Gradin et Erkki Likkanen.

Le cas de Paul van Buitenen, le fonctionnaire qui a transmis un rapport au groupe vert au Parlement et, ce faisant, relancé l'affaire, représente une dernière facette des effets de ce climat sur la "sortie» des affaires. Il permet également d'émettre quelques hypothèses sur les " agents révélateurs » et les raisons qui les conduisent à " déroger " au principe de réserve. Dans ce contexte de modification des cadres institutionnels et notamment de la frontière entre l'intérieur et l'extérieur, la frontière entre " exit, voice et loyalty " pour reprendre les catégories d'Albert Hirschman est moins claire qu'il n'y paraît au premier abord ${ }^{19}$. L'hypothèse n'est, en d'autres termes, permise que du fait du changement du jeu, il fait preuve de loyauté à l'égard de l'institution en sortant son rapport. Hollandais, Paul van Buitenen n'est pas un haut fonctionnaire. A 41 ans, il est de grade B3 et travaille au service du contrôle financier depuis 8 ans. Dans ce contexte, il n'est pas impossible que la rencontre entre la croyance dans l'exigence de la moralisation (auquel prédispose le tournant managérial et sa propre fonction) et le sentiment de la faillite de la Commission à la conduire (parce que trop politique) soit à l'origine de la remise de ce rapport. L'humeur de la dénonciation du Collège politique au motif même de la moralité qu'il prétendait porter et la porosité conjoncturelle de la frontière institutionnelle donne à penser que la remise de ce rapport ne représente pas un coût important mais qu'il est plutôt en phase avec le climat interne.

Ce climat fait du reste qu'il peut compter sur un ensemble de soutiens. Ces derniers s'expriment tout particulièrement à partir des sanctions dont il fait l'objet midécembre. Sur le plan interne, plusieurs tracts mobilisent les fonctionnaires et appellent au soutien de Paul van Buitenen - un compte bancaire est même ouvert. « La Commission tire sur le lampiste. Solidarité avec le collègue qui a dénoncé les fraudes » peut-on lire dans un tract Renouveau et Démocratie le 5 janvier. Ces tracts émanent tout d'abord des syndicats minoritaires mais qui se sont signalés par leur activisme lors des grèves du printemps dernier et ils témoignent d'une collusion entre les « libéraux » 
et «l'extrême gauche ». Mais ces soutiens se trouvent accentués lors d'une assemblée générale (statutaire) du personnel, tenue le 19 janvier où, à l'initiative des syndicats minoritaires, une résolution commune est votée à une très large majorité demandant la réintégration de Paul van Buitenen. Dans le contexte de la modification des frontières institutionnelles, ces soutiens sont également «extérieurs». Plusieurs articles paraissent sur "l'affaire van Buitenen" pour reprendre l'expression de l'agence Europe : dans un journal flamand en décembre, puis tout au long du mois de janvier dans l'ensemble de la presse. Van Buitenen fait même l'objet d'un ensemble de reportages télévisé, dont un sur M6. A l'extérieur comme à l'intérieur, ce sont les mêmes commentaires et preuves de soutien qui attestent de son " courage " et de son "caractère d'irréductible ", autant de qualificatifs qui le confortent dans son rôle et le mobilisent.

\section{Une remobilisation interne}

Les soutiens que peut escompter « l'agent révélateur » invitent à insister sur les usages internes des affaires. Au départ, la mise en exergue des affaires apparaît en effet comme un instrument du «troisième tour » des mobilisations du printemps 1998 et ce d'autant plus qu'en octobre, est annoncée la retenue sur salaire relative à la journée de grève ${ }^{20}$.

Aussi de septembre à janvier, les affaires et le règlement du conflit du printemps sont étroitement mêlés. À l'issue de la rencontre entre le président et les différents représentants des organisations syndicales, un tract commun aux organisations majoritaires définit la situation sous une forme qui renvoie assez nettement à ces " pressions » sur le Collège et son président : « les maladresses politiques du Collège et le mauvais exemple en général des commissaires et de leurs cabinets sont la cause du processus qui a conduit à une motion de censure au Parlement et aux insinuations des médias, sans compter le "ras-le-bol" du personnel ». Directement liées à des stratégies de politisation, les dénonciations de l'ultra-libéralisme refont leur apparition. Dans son tract du 18 janvier, l'Union Syndicale proche des socio-démocrates écrit: «La responsabilité du Collège actuel et de ceux qui l'ont précédé dans l'implication du privé dans des tâches de puissance publique est également très lourde ; la Commission et les autorités budgétaires, Parlement Européen en tête, semblent avoir organisé, d'un côté, la pénurie sciemment programmée des effectifs statutaires de l'autre côté, l'utilisation de crédits opérationnels pour introduire les intérêt privés dans les services de la Commission, jusqu'au sein de ceux chargés de la gestion des deniers publics. Ajoutons à cela, non pas l'absence mais la conception d'un dialogue social particulièrement stérile coupant la Commission de la réalité et de la compréhension directe du climat social, fonction essentielle dans une société moderne ». Ces propos ne sont pas le seul fait de l'Union syndicale. Ils sont plus largement nourris de la concurrence interne entre les syndicats. Celle-ci apparait sous une forme d'autant plus forte que s'ouvre la campagne pour l'élection des représentants du personnel qui a lieu au printemps.

Les effets de ce conflit entre le Collège et les fonctionnaires puis des concurrences entre les organisations syndicales ne sont pas anodins sur les possibles révélations. Non seulement ces tensions perpétuent le " climat ", mais elles nourrissent des mécanismes de requalification de la fraude qui, sans donner raison aux fraudeurs, donnent des raisons d'en imputer la responsabilité au Collège. Un sondage mis en circulation par Renouveau et Démocratie (document-tract du 4 novembre 1998) en est un bon exemple. 
En mêlant des questions sur la "réforme administrative " et sur les "dysfonctionnements, fraudes et irrégularités », ce document est un bon indicateur de la façon dont sont emboîtés le climat des grèves et celui des affaires. Mais ce sont surtout les opérations de qualification de la fraude qui sont à souligner ici. Les fonctionnaires doivent ainsi trancher entre des catégories du type défaillances techniques/défaillances morales qui recoupent assez explicitement la distinction administratif/politique. À la fin du même document, le syndicat montre très clairement le déplacement de la responsabilité des fraudes qu'implique un tel découpage. Il invite à « contraindre l'administration à s'engager dans une opération de moralisation en luttant efficacement contre les pressions politiques et les fraudes (...), poursuivre nos travaux d'enquêtes (...), interpeller l'administration pour l'obliger à justifier ses méthodes (procédures, enquêtes), définir les éléments précis de propositions et de revendications sur lesquels peut se porter la mobilisation du personnel ».

C'est en d'autres termes, l'origine même de la fraude qui est renversée. Cette dernière ne tient pas à l'incompétence (la thèse des "fonctionnaires underperforming ») mais aux pressions politiques. La concurrence entre les organisations syndicales aidant, les tracts interviennent du reste de plus en plus dans ce travail de requalification et plus largement de définition de la morale administrative (contre l'immoralité "politique » de la Commission). Dans un tract du 4 décembre intitulé «Opération mains propres : pour qui ? ", l'Union Syndicale écrit : " Au-delà de l'acte de ce fonctionnaire, se pose la question de l'existence de fraudes, de malversations et de la corruption, de leurs étendues réelles dans le tissu administratif, ainsi que de la capacité des organes de contrôle interne et externe de les détecter, puis de la volonté politique de la Commission d'en punir les auteurs reconnus. Une autre question, également importante, se pose quant à l'évaluation des notions de fraude, de malversation ou de corruption, notamment par rapport à celle de l'acrobatie, voire de l'irrégularité administrative. Le groupe des sages du PE devrait en finir avec les suppositions non fondées $"$.

Tout en dépossédant l'UCLAF du monopole de la qualification de la fraude, le tract vise le Conseil (organe politique par définition) et le Collège pour les acrobaties qu'ils favorisent. Mais il montre aussi les limites de la mobilisation et du jeu sur l'extérieur. En venant à l'encontre de l'image de pureté des fonctionnaires, le scandale vient toucher à quelque chose qui fonctionne comme un véritable capital collectif du groupe : l'incarnation de l'intérêt communautaire ${ }^{21}$. Aussi, est-ce à une clôture du jeu et notamment à l'égard de l'extérieur qu'appelle les représentants syndicaux majoritaires. Ce vœu de clôture vient du reste à la rencontre de la stratégie d'apaisement dans laquelle s'engage Jacques Santer à l'intérieur. Dans un message au personnel (sans date mais vraisemblablement de début février), Jacques Santer invoque ainsi toute une série d'arguments propre à refaire du lien en interne et à clore le jeu sur la frontière externe: "A cause d'une infime minorité de cas, l'opinion publique a focalisé son attention sur l'action de toute l'institution, au risque de jeter le discrédit sur l'ensemble d'un personnel loyal, honnête et qui consacre son énergie au service de l'institution. Le Collège refuse cette généralisation et se battra pour restaurer la confiance de l'opinion publique dans son personnel; Mais nous devons le faire tous ensemble. La force de l'exemple, l'excellence de notre travail, l'irréprochabilité de notre comportement, notre loyauté à l'institution, le respect des autres sont nos vrais atouts ». Il reste que cet appel à la clôture de l'institution sur elle-même intervient à un moment ou le jeu s'est déplacé de l'intérieur vers l'extérieur. 


\section{Politisation du scandale et effets institutionnels}

Bien qu'ils renvoient à d'autres équilibres, les développements interne et externe du scandale empruntent des chemins parallèles. C'est moins le tournant libéral de la Commission que son autre versant, l'accroissement de ses ressources politiques, qui est en cause. L'augmentation des pouvoirs de la Commission, sa transformation en "gouvernement de l'Union", et le recrutement de plus en plus politique des commissaires sont en effet autant de transformations objectives qu'ont bien en tête la plupart des acteurs. Mais ces transformations s'opèrent selon des modalités bien particulières : elles ne s'accompagnent pas des «servitudes " d'un gouvernement et demeurent en décalage avec les grilles de lecture politiques en usage. Aussi un ensemble d'acteurs, et au premier chef les journalistes et les parlementaires voient-ils leur position relativement dégradée, ou, comme présentant un « déficit " par rapport à la redistribution des pouvoirs que les réformes institutionnelles pouvaient leur faire anticiper.

Si telle est bien la structure des "frustrations externes" qui permettent le développement du scandale, elle ne porte pas sur tous les acteurs de la même façon. Aussi au départ, le scandale prend d'autres aspects que ceux d'une tactique «front contre front » : journalistes ou Parlement contre Commission. Dans cette perspective, la «sortie" des affaires puis le développement du scandale s'insèrent dans une succession de prises de position hétérogènes qui débouchent moins sur la démocratisation des institutions que sur la politisation des façons de jouer et sur un renforcement - a priori paradoxal - de la Commission dans sa revendication longtemps euphémisée d'être le gouvernement de l'Europe ${ }^{22}$.

\section{L'invention d'une humeur anti-institutionnelle}

Jacques Santer aurait dit de la responsabilité qu'elle est " une fleur rhétorique ». La répercussion de ce propos dans la presse est bien à l'image des frustrations politiques que peut faire naître la revendication de puissance par la Commission. Cette dimension s'observe tout particulièrement dans la convergence au départ assez involontaire d'un ensemble de stratégies journalistiques et politiques.

La révélation du scandale par la Lanterne, les enquêtes complémentaires menées par un groupe de journalistes ${ }^{23}$ puis par l'ensemble de la presse se caractérise moins par la volonté de faire exister un espace public européen que par la rencontre entre un processus d'autonomisation et des stratégies journalistiques animées par des motifs très différents. Pendant longtemps, les journalistes ont dans leur ensemble tenu lieu de relais d'information de la Commission européenne. La vocation fédéraliste commune des fonctionnaires et des journalistes tendait à fusionner le groupe des accrédités dans celui plus large des militants de l'Europe ${ }^{24}$. La Commission tenait par ailleurs les journalistes par une forme de "transparence stratégique » située dans le droit fil d'autres organisations internationales ${ }^{25}$. L'ouvrage de Jean de La Guerivière, Voyage à l'intérieur de l'eurocratie ${ }^{26}$, est assez emblématique de ces relations complexes entre la presse et la Commission. L'ancien correspondant du Monde montre bien les enjeux de l'accréditation, la fréquence des conférences de presse, l'abondance d'informations techniques ont pour effet de tenir les journalistes par les sources. A cela s'ajoute une 
autre technique assez classique et consistant à jouer sur le tableau d'honneur implicite que représentent la revue de presse pour rappeler à l'ordre les journalistes n'ayant pas relayé l'information.

L'accroissement du nombre des journalistes, l'arrivée de nouveaux journalistes se voulant plus "professionnels» et corrélativement la croissance des missions et de l'autorité de la Commission ont toutefois contribué à changer la donne ${ }^{27}$. Cette canalisation des flux d'information et leur inscription dans une seule dimension technique commencent à être de plus en plus dénoncées. L'ouvrage de Jean de la Guérivière en est un premier indice même si, publié en 1992, il demeure encore formulé sur un ton "diplomatique ». Ce n'est plus le cas du numéro du Monde diplomatique de juin 1996, dans lequel sont mis en exergue les techniques de "propagande » de la Commission, ce qui renvoie bien au sentiment d'une monopolisation de l'information éprouvé par un ensemble de journalistes ${ }^{28}$. Mais les tensions entre les journalistes et la Commission n'existent pas encore sous une forme explicite. Au départ de l'affaire, la connivence institutionnelle fait que les "problèmes » de gestion de la Commission ne sont pas «sortables» et du reste, les journalistes partagent les rationalisations des acteurs de la Commission. Le recours aux "sous-marins» - ces personnels de la Commission payés sur le budget des programmes communautaires au lieu de l'être sur un budget de fonctionnement en stagnation qui va devenir l'un des cœur des affaires fait, de ce point de vue, partie de ces demi-secrets qui soudent les « milieux informés ».

Aussi ce qui fait le développement de l'affaire tient moins à ce lent et continu processus d'autonomisation, qu'à la rencontre entre ce processus et des stratégies journalistiques assez hétérogènes. Trois types de stratégies vont permettre la sortie de l'affaire et, du même coup, l'accélération de l'autonomisation de journalistes : celui d'un journaliste marginal et spécialisé dans les "affaires" qui ont marqué la Belgique, celui de la revendication d'une position plus autonome et surtout ajustée aux évolutions politiques de l'Europe, et celui d'une presse allemande conjoncturellement portée à peser sur la Commission.

La Lanterne et le journaliste qui sort l'affaire tranchent ainsi avec les définitions de la pureté journalistique qu'ont en tête la plupart des commentateurs de la victoire de l'espace public européen. Situé dans le pôle de la droite chrétienne, le journal s'est distingué dans la critique des affaires en Belgique et plus généralement de la démocratie représentative. Le journaliste qui a sorti l'affaire, Jean Nicolas, est l'auteur de plusieurs ouvrages sur des thèmes à scandale dont les titres sont évocateurs : Les pédophiles sont parmi nous sur l'affaire Dutroux en 1997 ou Les protecteurs sur les proxénètes en 1998. Même s'il dément avec force son appartenance à l'extrême droite dans son livre, l'Europe des fraudes sorti immédiatement après la démission, le soupçon sur ses motivations reste partagé par un ensemble de journalistes ${ }^{29}$.

La reprise de ces « révélations » par un journal comme Libération pourrait étonner. Elle intervient pourtant dans un contexte - c'est le deuxième type de stratégie - où les affaires représentent une opportunité pour que s'impose une définition nouvelle du journalisme européen, plus indépendant vis-à-vis d'un Collège incarnant de plus en plus l'exécutif européen. Située dans son contexte bruxellois, cette opportunité vient à la rencontre de la mobilisation des fonctionnaires contre le Collège et il est assez probable que l'expressions de leurs frustrations soit venue donner du sens à celles des journalistes qui leur sont proches. La sortie de l'affaire présente en outre tous les avantages d'un «coup» sans risque, tant les journalistes peuvent tirer profit de la 
désolidarisation entre le Collège et les fonctionnaires de la Commission. En soutenant les premiers contre les seconds, les termes de l'échange domestique avec les fonctionnaires ne sont, au moins dans un premier temps, pas mis à mal par la dénonciation des affaires, s'ils ne les renforcent pas. Il est de ce point de vue assez frappant de constater que c'est selon des principes très proches de ceux que partagent les fonctionnaires européens que les affaires sont dénoncées : la bonne gestion, l'avenir de la construction européenne, le « déficit charismatique » du président Santer.

Dans le contexte journalistique national, les affaires offrent en outre une chance accrue de reconnaissance pour les journalistes spécialisés dans les questions européennes. Ces derniers ont en effet longtemps souffert d'une faible reconnaissance à l'intérieur de leur rédaction. Les sujets européens étaient réputés obscurs et n'intéressant pas le lectorat. Aussi les journalistes ont-ils dû conquérir leur position notamment par un déplacement du regard sur les coulisses, véritable instrument de pédagogie à la politique européenne. C'est par l'intermédiaire d'une rubrique sur les "coulisses de Bruxelles " que Jean Quatremer a réussi à stabiliser sa position à Libération ${ }^{30}$. D'une certaine façon, la révélation des affaires vient ici s'inscrire dans la suite «logique » de ce positionnement, tout en se conformant aux canons de l'excellence journalistique qu'incarnent l'image du journaliste d'investigation depuis les années trente et surtout l'affaire du Watergate ${ }^{31}$. Au demeurant, les articles de Libération s'inscrivent bien dans le tournant à la fois apolitique et moral - il s'agit d' "affaires » européennes - du journal analysé dans la thèse de Philippe Juhem ${ }^{32}$.

Cette adéquation à l'égard des logiques du marché national s'observe de façon différente dans le cas de pays comme l'Allemagne ou l'Angleterre. Le cas allemand apparaît de ce point de vue tout à fait exemplaire. A la différence des journalistes français, les journalistes allemands spécialisés sur les questions européennes n'ont pas, dans leur ensemble, à «faire » leur position. Ils sont les plus nombreux et la politique européenne fait partie des rubriques reconnues. Mais il semble que cette reconnaissance joue dans le sens d'une homologie entre leur prise de position et celle de l'État. La "position» allemande dans les négociations de l'agenda 2000 de la revendication d'un budget rééquilibré et la critique des gaspillages de la Commission fait l'objet d'un large consensus. Elle a fait partie des thèmes de campagnes communs des forces politiques allemandes et du reste, cette "pression " allemande est assez durement ressentie au sein de la Commission. Aussi ce n'est pas vraiment étonnant si à l'origine des révélations se trouvent un journaliste d'ARD puis d'autres du Frankfurter Allgemeine Zeitung. Un article de Wirtschaft Woche de novembre 1998 est assez révélateur des thèmes qui animent la critique de la Commission ${ }^{33}$. Les salaires disproportionnés des fonctionnaires sont exhibés. Les grèves contre Likkanen et l'introduction de ses critères de performance sont ensuite raillées sur le mode de l'irréalité des fonctionnaires. De même est dénoncé un ensemble de faits mineurs mais considérés comme emblématiques de la mauvaise gestion de la Commission, comme les vols de classes économiques remboursés au prix de la classe affaire, les trucages des feuilles de frais, etc. Les exemples pourraient être multipliés notamment en ce qui concerne la presse anglaise ${ }^{34}$.

Si ces positions apparaissent au départ difficilement conciliables, elles vont bientôt se rencontrer du fait du développement de l'affaire au Parlement. Là encore une lecture ex-post pourrait conduire à voir la dénonciation du scandale comme l'aboutissement logique de l'autonomisation du Parlement. De fait dans un état antérieur du jeu, le Parlement européen se caractérise plutôt par des mécanismes de consensus interne et 
de solidarité relative avec la Commission facilités par le fait que le Parlement européen demeure un champ périphérique ${ }^{35}$. A y regarder de plus près, l'accélération du scandale au Parlement tient pourtant moins à un processus d'émancipation collective qu'à des logiques de concurrences internes. Bien que dotés de pouvoirs accrus, la structure du jeu parlementaire n'a pas véritablement changé, mais le quasi-monopole des deux grands groupes que représentent le PSE et le PPE est, là encore, l'origine d'un ensemble de « frustrations ».

Dès les révélations de l'affaire, c'est ainsi la député du groupe des Verts Edith Muller, bientôt appuyée par la chef de son groupe, qui est la plus virulente et qui la première au début du mois de septembre - qualifie les «affaires » de "scandale ». Membre de la commission de contrôle budgétaire (la COCOBU), elle s'est distinguée par des prises de positions assez fermes contre l'opacité de la gestion à la Commission et c'est à elle qu'a recours le journaliste de La Lanterne lorsqu'il est en possession des "dossiers » livrés par Claude Perry. C'est ensuite autour de la COCOBU que se développe l'affaire au Parlement. Celle-ci occupe une position qui est, là encore, bien à l'image de la façon dont peuvent peser les «frustrations" au Parlement. Lieu par définition d'une orthodoxie budgétaire assez en phase avec la volonté affichée de la Commission, la COCOBU ne représente pas la commission parlementaire la plus prisée. Placée sur un pôle technique qui s'oppose au pôle politique des "grandes" commissions, comme celle des affaires étrangères - l'ex-commission des affaires politiques - ou de la réforme institutionnelle, elle ne bénéficie pas non plus du prestige de celle du budget ou de la commission juridique. Ce ne sont pas les députés les plus en vue qui la composent et la place des groupes minoritaires comme les libéraux et les verts y est importante. Elle est enfin - et le fait à son importance dans la tournure politique qui sera prise par les événements - présidée par une allemande du PPE, au moment même où la pression allemande sur le budget de la Commission est forte et où les commissaires les plus en cause sont socialistes.

Aussi est-ce moins dans un affrontement front contre front que dans cet éparpillement $\mathrm{du}$ jeu que prend forme le scandale. Car pour lui faire face, les acteurs vont puiser dans les savoir-faire politiques qu'ils possèdent, et ce faisant, engendrer une dynamique dans laquelle les affaires vont représenter le « point focal $»^{36}$ d'un ensemble de jeux-test politiques.

\section{Politisation politique et jeux-test institutionnels}

Dans son numéro du 4 et 5 janvier, l'éditorialiste de l'agence Europe écrivait pour le déplorer: "le déroulement visible des événements cache des manœuvres, des intentions pas toujours avouées, parfois même des manipulations et des intrigues ${ }^{37}$. La formule dit bien que la situation favorise des calculs politiques de plus en plus nombreux. Il reste que c'est moins leur nombre que leur relative nouveauté dans le jeu politique européen qui marque la déception, la surprise ou la jubilation des acteurs. Nourris par la rencontre entre les tentatives de «sortie de crises » envisagées par les Commissaires et les mobilisations de plus en plus convergentes des parlementaires et des journalistes, ces calculs se traduisent tantôt dans des oppositions droite/gauche, tantôt dans des luttes de courants internes aux groupes et tantôt dans une opposition entre "l'exécutif » et «le législatif » qui, dans ce jeu très incertain, vont offrir aux différents protagonistes des opportunités d'une remise en ordre institutionnelle. 
Bien qu'hétérogènes, les prises de position convergentes des journalistes et des parlementaires verts, en particulier, ne sont pas sans effets. Erving Goffman a bien montré ce qui se produit lorsque se brisent les cadres de l'interaction : le fou-rire, les pleurs, la violence ${ }^{38}$. Cette rupture de cadre peut se lire dans les pleurs publics d'Emma Bonino, et ses menaces de porter plainte contre le Financial Times (Libération, 14 octobre 1998). Mais elle peut se lire aussi dans la violence des ripostes d'Edith Cresson.

Il faudrait faire ici l'inventaire complet des stratégies visant à conjurer la «crise ». Le journaliste Jean Nicolas en livre un ensemble comme la désinformation, la propagande, les pressions et les manipulations secrètes, qui renvoient bien à l'inflation des calculs politiques et à leur inscription dans des schèmes "classiques" du jeu politique national. Pour ce qui est des tactiques publiques, les réactions d'Edith Cresson aux révélations puis à leur reprise se situent ainsi dans la droite ligne d'un comportement politique lui aussi «classique ». Les poursuites judiciaires à l'égard de Libération et la publication d'un droit de réponse indiquent bien ce ton, proche de l'intimidation mais sur son versant légitime, qui est pratiquée dans l'ordinaire des affaires nationales. En consistant à faire valoir son souci de transparence et en offrant de l'information au moment même de cristallisation des affaires, l'interview donnée au Monde deux jours après le droit de réponse à Libération représente, pour sa part, une technologie de neutralisation politique par excellence, même si ces tentatives se déroulent dans un ensemble où les protagonistes sont suffisamment indépendants pour que la riposte ne soit pas efficace à $100 \%{ }^{39}$.

A cela s'ajoute, mais plus tard, la dénonciation du complot. Cette dernière peut de ce point de vue apparaitre comme autre chose que le produit de schèmes paranoïaques ${ }^{40}$. Opposant une autre révélation à celle des affaires, la dénonciation du complot renvoie bien à cette situation de « crise de régime » telle qu'elle est perçue par les acteurs et, ce faisant, à des schèmes d'interprétation et d'action historiquement éprouvés ${ }^{41}$. Elle correspond aussi à une stratégie politique en voie d'expérimentation sur le terrain européen : E. Likkanen y a recours au moment des fuites du concours, Jacques Santer parle de " chasse aux sorcières ». Surtout, elle apparaît ici particulièrement ajustée à la complexité du jeu. Du fait des alliances précaires et de l'incertitude forte qui pèse sur les relations internes aux institutions européennes, cette stratégie présente de fait toutes les caractéristiques d'une mise en abîme et rendant illisible la situation. C'est le cas pour les journalistes qui ne maîtrisent pas complètement les positions et les logiques journalistiques des autres pays que les leurs. C'est le cas aussi des relations entre les différents représentants nationaux au moment même où les relations sont tendues entre l'Allemagne et la France sur la négociation de l'agenda 2000.

Si cette politisation des savoir-faire concerne les ripostes de la Commission en direction de la presse, elle concerne aussi les relations avec le Parlement. De ce point de vue, la réaction de Jacques Santer pourrait bien relever d'une "erreur tactique», pour reprendre les termes de l'éditorial de F. Riccardi dans le bulletin quotidien Europe qui renvoient bien, là encore, à la forte présence des calculs. Au moment du vote de la décharge budgétaire en décembre, ce dernier ne veut interpréter ce vote comme autre chose qu'une défiance, et c'est lui qui entame le premier la "montée aux extrêmes » par l'intermédiaire d'une lettre au président du Parlement. À ce geste répond alors une innovation institutionnelle réinterprétée comme un moment "chaud" dans la concurrence entre les deux institutions: le Parlement refuse d'accorder la décharge budgétaire par 270 voix contre 230 et quelques abstentions. 
Cette position de surplomb va pourtant s'infléchir à partir de novembre, mais là encore dans des cadres qui relèvent $d u$ test d'une nouvelle tactique politique: la parlementarisation du jeu institutionnel. Toutes les relations entre le Collège et les parlementaires ne se réduisent pas à un face à face. Là encore, la motion de censure doit être située dans son contexte. Au départ, elle ne correspond pas exactement à un acte démocratique de contrôle, mais plutôt à une transaction passée entre le président Santer et les parlementaires, et en particulier les socialistes, pour re-légitimer l'institution. Pour la Commission, l'intérêt est assez clair : il s'inscrit non seulement dans le contexte des scandales mais aussi d'un ensemble de négociations dans lequel la Commission est engagée -avec les représentants des Etats membres, au Conseil. Dans cette perspective, et sans doute aussi pour ramener l'enjeu dans le cadre des institutions les plus européennes ${ }^{42}$, la sortie de crise passe par l'innovation institutionnelle que représente ce vote de confiance. Cette procédure n'existe pas. Aussi c'est à l'aide d'une motion de censure déposée par le groupe socialiste (majoritaire) - ce qui lui permet au passage de renforcer sa position ${ }^{43}$ - qu'elle peut s'opérer.

La façon dont Jacques Santer s'en explique dès novembre dans une réunion avec les organisations syndicales et le discours de Pauline Green au moment du vote sont assez claires ${ }^{44}$. Et du reste c'est dans cette veine que s'inscrit le discours du président Santer du 11 janvier. En invitant à juger la Commission sur «son action d'ensemble et son bilan réel en tant que Collège ", le discours de Jacques Santer est un discours de vote de confiance: il s'inscrit dans huit points généraux qui non seulement minorent les affaires, mais représentent un véritable programme politique (sur l'emploi, l'Euro, l'élargissement, la réforme institutionnelle). Cette inflexion parlementariste s'observe dans l'appel aux réflexes politiques des parlementaires : « La Commission n'a pas voulu faire pression sur les parlementaires - énonce Jacques Santer lors de son discours. Je connais moi-même trop bien la vie parlementaire ici mais aussi dans mon pays. Mais la Commission sait qu'elle a besoin de la confiance ».Une fois la motion de censure écartée, Jacques Santer prend la parole :» votre message à la Commission est clair, je l'ai reçu cinq sur cinq. Je prends à cœur les critiques exprimées, j'accepte la création d'un comité des sages et ses recommandations seront suivies d'effets ». Et Jacques Santer de conclure: "le Parlement européen a affirmé avec force son autorité et renforcé son rôle de contrôle démocratique ». Tout indique - on y reviendra - que Jacques Santer attend de ce revirement qu'il mette fin à ce qui est de plus en plus appelé une "crise institutionnelle ». Partout ailleurs qu'au Parlement, la définition de la situation est celle du lancement de l'Euro ${ }^{45}$ et il est assez probable que la nomination du groupe des sages ait été perçue comme une technologie politique de «maîtrise des crises » suffisante au même titre que la transformation de l'UCLAF en Olaf, organisme indépendant ${ }^{46}$.

Il reste que cette conciliation intervient trop tard. Plus encore, en soulignant l'alliance entre le Collège et le groupe majoritaire, elle tend à exacerber les oppositions. Ce changement du jeu s'observe tout particulièrement dans le débordement - au sens sportif du terme - qui s'opère par l'intermédiaire de la seconde motion de censure, véritable rupture par rapport aux traditions de compromis. Déposée par Hervé FabreAubrespy, député villieriste français, maître des requêtes au Conseil d'État et ancien conseiller technique au cabinet de Charles Pasqua entre 1986-1988, la seconde, en revanche, se présente comme la « vraie » motion contre la « vraie-fausse » motion des socialistes, autant de termes qui renvoient, en France du moins, à d'autres scandales 
politiques. Elle est par ailleurs le fruit d'une alliance paradoxale conclue entre villiéristes, libéraux et verts qui montre bien qu'elle s'inscrit contre la motion dominante, et réputée jouée d'avance, du PSE. Cette composition montre bien que le " coup " part des marges et sur les marges, mais elle donne aussi à penser à la façon dont se superposent, ici encore, logiques européennes et nationales. En France, la réunion du Parlement pour la révision de la Constitution devant permettre la ratification du traité d'Amsterdam a lieu quatre jours après le vote de cette motion de censure. De même, la position ferme des Allemands est en étroite relation avec celle des journalistes allemands et peut tout à fait s'interpréter comme une préparation des élections européennes. Cependant, elle intervient aussi à un moment où l'Allemagne prend la présidence dans les négociations importantes autour de l'agenda 2000. Aussi, pour les députés allemands, la motion de censure représente une occasion de faire d'une pierre deux coups, le premier dirigé contre la Commission le second contre la France - par l'intermédiaire d'Edith Cresson -- à un moment où celle-ci représente son principal obstacle.

Cette transformation du jeu se donne encore à voir dans les résultats du vote de cette seconde motion et dans ses interprétations. Ceux-ci montrent, de façon analogue à ce qu'on observe au sein de la Commission, une opposition très forte entre les pays du Nord et ceux du Sud. Si tous les Allemands ont voté oui, tous les Espagnols, les Portugais et les Italiens ont voté contre. À cela s'ajoutent les divisions internes et la politisation des discours et des prises de positions. Tout au long des débats, les arguments « électoraux » sont constamment évoqués. Les échanges prennent du reste la forme de jeux-test politiques où se rodent des alliances possibles ou encore l'identité des marques politiques. Pour donner quelques exemples, Nicole Fontaine et un ensemble de députés ayant voté non à la seconde motion dénoncent « l'esprit partisan et les intérêts nationaux » dans un registre européen qui préfigure directement ce que sera la campagne de l'UDF. Au moment de la nomination du groupe d'experts, Hervé Fabre-Aubrespy écrit un courrier à José Maria Gil-Roblès pour lui proposer de nommer Antoine Gaudino, le révélateur de l'affaire Urba ${ }^{47}$.

Cette transformation du jeu s'observe enfin dans la distance que les journalistes prennent de plus en plus collectivement. Cette distance se marque dans la fonction de contrôle démocratique qu'ils entendent incarner, et ce d'autant plus que l'Association de la Presse Internationale a pris position contre les manœuvres de la Commission ${ }^{48}$. L'article de Jean Quatremer, du 31 janvier 1999, est ainsi parfaitement exemplaire de la façon dont les journalistes font valoir leur vigilance. Il relate en effet une bévue de la Commission qui laisse s'afficher une note regrettant « la prise de la salle de presse par les journalistes d'investigation » et incite à "une dose de cynisme pour tenir les confrontations » avec les journalistes. Cet événement fait là encore grand bruit et prend toutes les caractéristiques d'une autre "affaire dans l'affaire ", à un moment où les effets de la nomination du comité des sages se sont tassés et où la plupart des représentants s'accordent à reconnaître que les jeux sont faits.

Mais indépendamment de cette posture, les journalistes ont recours à des schèmes d'interprétations de plus en plus proches de ceux qu'ils prennent ou qu'ils ont historiquement pris dans l'espace national. C'est le cas lorsqu'ils s'engagent sous la forme de la lettre ouverte, format comparable à celui du «J'accuse » d'Emile Zola ou des différentes lettres ouvertes par lesquels les journalistes ont revendiqué leur autonomie sous la Cinquième République, comme dans cet article de la page débats intitulé «Vive l'eurocensure » co-signé par J. Quatremer (11 janvier 1999). Mais c'est 
surtout le cas dans les grilles de lecture auxquelles ils ont recours. Belle illustration conjointe des mécanismes de frustration et de politisation des catégories qui s'opèrent, les journalistes sont d'autant plus conduits à voir dans Jacques Santer un anti-Delors, voire un "homme faible", qu'ils ne le jugent pas à la hauteur de la définition du "gouvernement " qu'ils ont en tête. À cela s'ajoute la répercussion des calculs sur la succession de Santer. Le nom de Romano Prodi est ainsi avancé dès novembre 1998 soit cinq mois avant la démission et plus de huit mois avant le remplacement ce qui tranche avec la fois précédente. De même c'est dans des catégories éminemment politiques que sont interprétées les possibles nominations, comme celle d'Oscar Lafontaine, ce qui là encore rompt avec les pratiques en usage lors des précédentes nominations à la Commission.

On voit mieux ainsi comment ont peu à peu pris forme ces nouvelles façons de penser ou d'agir au sein des institutions européennes. Celles-ci n'ont du reste pas été sans effets. De façon toute politique, tous les acteurs, y compris les plus proches d'une solution de compromis comme l'ont été à un moment Pauline Greene et Jacques Santer, ont été collectivement conduit à invoquer de plus en plus "l'opinion européenne » et, surtout, envisager une sortie de crise dans les canons du jeu parlementaire. Aussi, à partir de février, la démission apparaît-elle pour beaucoup comme la seule issue.

\section{Jugements et consolidation institutionnelle}

La politisation qui résulte de la crise et de cette succession de coups peut se lire d'une autre manière: dans la position centrale prise par la Commission dans le dispositif institutionnel, et ce au détriment d'un Conseil largement absent de ces débats. L'hypothèse est en effet permise que le scandale a moins nui à la Commission qu'on l'a dit. En devenant un " point focal », la Commission s'est trouvée placée au centre du jeu et d'une certaine façon elle a renforcé la position de "centre de force » qu'elle tend déjà à occuper au sein des institutions européennes ${ }^{49}$. Dans ce moment qui aurait dû faire la part belle au Conseil (agenda 2000, réforme institutionnelle, et nomination de la future Commission), les affaires semblent moins avoir été l'instrument de la victoire de la démocratie que d'un déplacement du jeu vers les institutions les plus européennes, bon remède temporaire aux frustrations observées.

Cette position renforcée peut se lire, par contraste, dans la démission même de la Commission. Loin de n'être que la suite logique du rapport des sages, la démission représente un coup destiné à sauver la face à Edith Cresson tout en escomptant une rapide remise en scelle de la plupart de ses membres. Il faut, de ce point de vue, rappeler que le Commissaire français a constamment signifié son refus de démissionner et notamment en faisant valoir les soutiens conjoints de Jacques Chirac et de Lionel Jospin. Aussi l'issue que représentait la possibilité que la Cour de Justice déjuge nominalement les commissaires incriminés relevait d'une stratégie à haut risque, voire impossible. Mais, dans le même temps, la démission ne semble pas représenter un coup par défaut pour la plupart des commissaires. Elle tient aussi dans la relative confiance de Jacques Santer et d'une partie des membres du Collège d'être reconduits dans leur fonction une fois les «brebis galeuses »- c'est le terme employé off par des commissaires - écartées. La divulgation à l'extérieur de ce terme n'est qu'un exemple de ces stratégies - là encore très politiques - visant à rendre visible le partage entre le pur et l'impur et donc à renforcer la légitimité des bons commissaires. Les déclarations de Jacques Santer aux lendemains de la démission sur sa volonté de poursuivre le travail engagé et 
sur la réussite de « l'opération mains propres » sont assez éclairantes sur les chances de succès qu'il escompte. La position d'un ensemble de pays - c'est le cas de la France semble donner à penser qu'une telle solution n'est pas à écarter.

Les Commissaires sont d'autant plus en mesure de croire à la confiance dans la « force institutionnelle » de la Commission que celle-ci s'est trouvée renforcée dans les luttes. Il est en premier lieu frappant de constater que la démission n'est pas l'effet d'un « jeu tendu », mais qu'elle est passée par une " instance d'exécution » extérieure, le comité des sages, et qui plus est nommé par un compromis entre le Parlement et la Commission elle même ${ }^{50}$. En d'autres termes, l'autorité symbolique dont la Commission est investie - en tant que " gardienne des traités » mais surtout détentrice du monopole de l'intérêt communautaire - apparaît telle qu'il est presque impossible pour la plupart des parlementaires de penser à la renverser. Lorsqu'à la veille de la remise du rapport des sages, les différents groupes dramatisent la situation et font monter la pression, les arguments sont échangés par presse ou communiqués interposés.

Mais, surtout, tout au long du développement du scandale, les accusations de la Commission se doublent d'un ensemble de stratégies visant à la préserver en rappelant son rôle dans l'unification de l'Europe et en renvoyant sur le pôle anti-européen toute tentative de lui porter atteinte. Il passe également par un ensemble de démonstrations symboliques fortes, comme le recours aux grandes figures de la construction européenne. Le 11 janvier, le jour où il tient son discours « gouvernemental » devant le Parlement, Jacques Santer participe à la présentation d'un livre sur Walter Hallstein. $\mathrm{Au}$ moment fort de la crise, la Commission en direct fait sa « Une » sur Jean Monnet.

Mais, au-delà, s'instaure tout au long de l'affaire comme une pédagogie du jugement qui renforce une symbolique des institutions européennes qui fait de la Commission le cœur de la construction de l'Europe. A l'extérieur, les arguments développés par l'agence Europe sont de ce point de vue très significatifs : "Les critiques aux lacunes, voire aux fautes de la gestion budgétaire se sont transformées, au cours des mois, en une remise en cause globale de toute l'action de la Commission européenne, à la plus grande joie des adversaires de la construction européenne elle-même.[...] Mais il est nécessaire de garder le sens des proportions. Un jugement politique sur la Commission doit se fonder sur l'évaluation de son activité par rapport à l'Europe, sur la valeur de ses initiatives, son comportement face au Conseil et aux autres institutions, sa capacité de proposer ce qu'il faut au moment où il faut et de savoir le défendre si nécessaire devant les états membres ${ }^{51}$.

Les prises de position au moment de la censure sont tout aussi exemplaires. C'est tout particulièrement le cas à gauche. Si elle augmente la capacité de négociation du PSE, la proposition de la fausse motion de censure est destinée à relégitimer la Commission. Mais indépendamment de cette tentative, les discours tenus représentent des pratiques de consolidation institutionnelle. Dans le climat de la création de compromis et de la relative confiance à l'égard du vote du PPE, le socialiste (membre du PDS italien) lance un appel aux composantes pro-européennes pour qu'on arrête une crise sans précédent. Si la dramatisation relève des stratégies politiques de sortie de crise, son discours porte bien sur la "valeur» que représente la Commission parmi les institutions européennes : "c'est une chose que de demander à la Commission de corriger ses défauts de gestion et de mettre en route sa propre réforme, c'en est une autre que de provoquer une crise et favoriser la diminution de son autonomie, une sorte de mise sous tutelle qui la réduise à une sorte d'agence du Conseil $\aleph^{52}$. 
Mais c'est aussi le cas à droite. Dans le climat de politisation qui marque les affrontements, les catégories politiques viennent brouiller les possibilités d'une opposition frontale, même en provenance de « la droite ». Bien que la Commission soit plutôt à dominante sociale-démocrate, elle est dirigée par un démocrate-chrétien. Aussi l'opposition porte moins sur le Collège des commissaires que sur les deux socialistes Manuel Marin et Edith Cresson. Il est assez frappant que le rapport Brock (député PPE) sur la réforme institutionnelle qui sort précisément au moment des débats sur la censure reprenne un ensemble de propositions de Jacques Delors, telles qu'elles apparaissent dans la presse depuis mai 1998, puis au début janvier 1999, visant précisément à renforcer la position gouvernementale de la Commission. C'est bien cette transformation de la Commission en gouvernement en relation plus étroite avec le Parlement, et ce faisant le déplacement du centre de gravité du triptyque institutionnel vers des formes plus proches des jeux politiques nationaux, qu'ont en tête la plupart des députés, et non sa délégitimation. Ce n'est pas véritablement étonnant que ce rapport soit critiqué sur les marges villiéristes par Georges Barthu qui dénonce «la transformation progressive de la Commission en une sorte de gouvernement $»^{53}$.

$\mathrm{Si}$ ces processus de consolidation institutionnelle mériteraient plus ample développement, la nomination de Romano Prodi à la tête de la Commission n'infirme en tout cas pas ce processus plus général de transformation de la Commission en gouvernement de l'Europe. Il reste qu'avant d'en venir aux effets, ces quelques lignes de conclusion voudraient d'abord porter sur les processus dans lesquels a pris forme le scandale. Ceux-ci sont à comprendre dans une double dimension. Ils correspondent d'abord à des processus de moyen terme et qui tiennent à la redistribution des ressources politiques internes aux institutions. Marc Abélès a bien montré la frustration structurelle des parlementaires européens ${ }^{54}$. Elle se double ici du décalage entre les attentes suscitées par le traité d'Amsterdam et, en l'absence de ratification, l'exclusion des processus capitaux se jouant entre la Commission et le Conseil. Et surtout elles en rejoignent d'autres qui portent sur les groupes minoritaires au Parlement et - c'est un point de départ - celle des fonctionnaires européens. Ces frustrations ne fonctionnent cependant pas comme un facteur extérieur. Le scandale naît en effet du succès des mobilisations qui s'opèrent, c'est-à-dire dans l'action et surtout dans des tentatives, ici réussies, d'accorder des stratégies très différentes et, chemin faisant, de réduire la complexité du jeu. De ce point de vue, on peut faire l'hypothèse que beaucoup de choses se sont jouées dans la mise en accord des temporalités très différentes dans lesquels les acteurs sont ici collectivement engagés c'est un des problèmes que pose l'existence d'un espace institutionnel européen. Sans en faire un facteur, il y a eu comme une tentative d'harmoniser ou de faire «converger" les calendriers très différents dans lesquels ils évoluent: calendriers nationaux, des groupes parlementaires, du Conseil et des négociations, de la Commission, des journalistes qui préparent les élections européennes. La mise en parallèle de ceux-ci montrent comme des zones de rencontres et de turbulence et, d'une certaine manière, les affaires ont favorisé l'émergence d'un point de coordination et d'ajustement entre ces différentes temporalités et ce n'est pas là le moindre des signes de la construction, au moins éphémère, d'un espace politique.

De ce point de vue, le scandale pourrait bien représenter un instrument de mis en ordre du jeu politique. L'un de ses effets a du reste été ici d'importer un ensemble de schèmes politiques éprouvés dans les différents espaces nationaux (opération "mains propres ", dénonciation du complot, stratégie jusqu'au-boutiste, usages politiques des affaires), 
permettant aux acteurs de remettre de l'ordre dans cet univers complexe et, surtout, de « jouer » ensemble. C'est de cette forme d'« excitation collective», pour emprunter au Durkheim des formes élémentaires de la vie religieuse ${ }^{55}$, qu'est né l'emblème de la victoire de la démocratie européenne. Reste à savoir quelle sera la pérennité de ses effets. Seule une sociologie politique de l'Europe nourrie par l'étude empirique des acteurs, des enjeux qui les animent, des perceptions qu'ils en ont, ou encore de la façon se construisent leurs interdépendances, permettra de le dire.

\section{NOTES}

1. Jean Quatremer, Libération, 16 mars 1999. Le terme de "grand déballage » est une révérence au titre du journaliste qui a révélé l'affaire fin août 1998.

2. C'est le titre d'un article du Monde, 19 mars 1999.

3. Sur les usages de la figure de l'eurocrate, cf. notre article dans V. Dubois et D. Dulong, La question technocratique, PUS, coll. « sociologie politique européenne », 1999.

4. Cette contribution est une note d'étape liée à une recherche plus générale sur la sociologie des euro-fonctionnaires et plus généralement des institutions européennes. Proposée avant la démission de la Commission, elle se donnait principalement pour but de comprendre la façon dont les «crises" de la construction européenne participent à son institutionnalisation. La difficulté d'avoir recours à des entretiens une fois la «crise passée » a conduit à privilégier un ensemble de sources principalement écrites. Il s'agit, pour la période d'août 1998 à mars 1999, de l'ensemble des tracts syndicaux émis au sein de la Commission et de l'ensemble des numéros de la Commission en direct, l'organe interne des fonctionnaires. Il s'agit également de l'intégralité de 7 jours d'Europe, l'organe de la représentation en France de la Commission européenne, et du bulletin quotidien de l'Agence Europe (BQE)A cela s'ajoutent les communiqués de presse des groupes politiques au Parlement disponibles sur Internet, la revue de presse systématique du Centre d'Information sur les Institutions Européennes de Strasbourg croisée par une recherche systématique dans le Monde et les Dernières Nouvelles d'Alsace. Un ensemble d'entretiens informels viennent compléter les sources suscitées. Je remercie Marine Delasalle pour sa lecture de la première version de ce texte.

5. Sur les scandales, cf. M. Dobry, Sociologie des crises politiques, Paris, Presses de la Fondation Nationale des Sciences politiques, 1986 p.291; Alain Garrigou, «Le boss, la machine et le scandale ", Politix, $n^{\circ} 17$, premier trimestre 1992 et Pierre Lascoumes, Elites irrégulières. Essais sur la délinquance d'affaires, Paris, Gallimard, 1997.

6. Alain Garrigou, « Le boss, la machine et le scandale », op.cit.

7. Sur l'UCLAF et la lutte de politique anti-fraude cf. notre article, «Les instrumentalisations de la morale : lutte anti-fraude, scandales et nouvelle gouvernance européenne », dans J.-L. Briquet et $\mathrm{P}$. Garraud, Juger la politique, Rennes, Presses universitaires de Rennes, à paraître en décembre 2000. Pour des données sur son organisation à la veille des scandales, cf. le rapport de la Cour des comptes, rapport spécial n8/98.

8. Howard Becker, Outsiders. Etudes de sociologie de la déviance, Paris, Métailié, 1985 (1ère ed.1963).

9. Le Monde Diplomatique emprunte beaucoup à un numéro antérieur de European Voice, 21 mars 1996 ; François d'Aubert, Coup de torchon sur Bruxelles, Paris, Fayard, 1999. 
10. Sur ce point, cf. Marc Abélès, Irène Bellier, Maryon Mac Donald, Approche anthropologique de la Commission européenne, Commission européenne, Bruxelles, 1993.

11. Sur les groupements hiérocratiques, cf. Max Weber, Economie et Société, Paris, Press-Pocket, 1995, pp.96-100. Sur les formes hiérocratiques de la construction européenne, cf. notre communication « La richesse d'un groupe : les portraits des euro-fonctionnaires dans la rubrique "people » de la Commission en direct », lors de l'atelier sur la Commission européenne, animé par Eve Fouilleux et Cécile Robert, communication au Congrès de l'Association Française de Science Politique, Rennes, septembre 1999.

12. Sur ces points, cf. en particulier, George Ross, Jacques Delors and European Integration, New York, Oxford University press, 1995 et Helen Drake, Jacques Delors, Londres, Blackwell, 2000.

13. Sur les commissaires, cf. l'article d'Andy Smith dans ce numéro.

14. La notion de "climat» mérite sans doute quelques lignes d'attention. La description apparemment neutre du climat est tout d'abord l'un des instruments grâce auxquels les acteurs pèsent sur la situation tout au long du scandale. La liste est inépuisable : « climat délétère de fin de règne » énonce un tract de l'Union Syndicale (4 décembre), «climat délétère » (Le Monde 5/4 octobre), " ambiance crépusculaire », dit Jean Quatremer (Libération, 12 janvier). Mais son usage renvoie aussi à une modification des liens et des relations objectives au sein de la Commission. La description du climat n'apparaît en effet pas avec le scandale. Elle prend sa source dans les grèves d'avril 1999 - sur lesquelles nous reviendrons - et correspond à un ensemble de tensions entre le « haut » et le «bas », le Collège et les cabinets contre les directions générales, les hautsfonctionnaires contre les fonctionnaires «tout court», le Nord contre le Sud, les sociodémocrates et socio-chrétiens contre les ultra-libéraux, les « jeunes » contre les « vieux », autant de clivages qui vont ici contribuer à «ouvrir le jeu»Instrument de lutte, la qualification du climat n'en a pas moins des effets de réalité. En donnant du sens à ces modifications, elle finit par définir la situation et, ce faisant, par jouer sur les anticipations et les calculs des protagonistes.

15. Sur Erkki Likkanen, cf. l'article de Niilo Kauppi dans ce numéro.

16. Sur ce point, cf. notre article, «Une mobilisation formatrice : les eurofonctionnaires contre la réforme du statut (printemps 1998) » in Renaud Dorandeu et Didier Georgakakis, L'Europe sur le métier. Acteurs et professionnalisations de l'Union européenne, Strasbourg, PUS, à paraittre en janvier 2001. Sur la réforme de la Commission, cf. également Michele Cini, « Leadership and Culture : The Commission President and Commission Reform 1995-2000», Working paper, Loughborough, Workshop, 23 juin 2000.

17. Pierre Lascoumes, Elites irrégulières, op.cit.

18. Jean Nicolas, L'Europe des fraudes, PNA, Bruxelles, 1999.

19. Albert Hirschmann, Exit, Voice and Loyalty, Cambridge, MA, Harvard University Press, 1970.

20. The European Voice, 10-16 septembre 1998.

21. Sur cet aspect, outre les travaux de Marc Abélès et Irène Bellier, déjà cités, cf. également Andy Smith, L'Europe au miroir du local, Paris, L'Harmattan, 1996, L. Cram et C. Lequesne, «La Commission, entre autonomie et dépendance", Revue française de science politique, 46/3, pp. 398-399.

22. Sur scandale et consolidation constitutionnelle, cf. Alain Garrigou, « Le président à l'épreuve du scandale. Déstabilisation apparente et consolidation fonctionnelle », in Bernard Lacroix et Jacques Lagroye, Le Président de la république, usages et genèses d'une institution, Paris, Presses de la Fondation nationale de science politique, 1992.

23. Les remerciements qu'adresse Jean Nicolas dans son ouvrage sont un indicateur au moins partiel de l'existence d'un noyau dur parmi les journalistes Jean Quatremer de Libération, Hartwig Nathe (Focus), André Riche (Le Soir), Stéphan Grey (Sunday Time), Udoi Lielischkies (ARD).

24. Sur ce point, cf. la contribution de Caroline Schickel, au colloque Les métiers de la construction européenne, op.cit. et $\mathrm{O}$. Baisnée, «Can Political Journalism Exist at the European 
Level?", communication à l'atelier «Political Journalism: New challenges, New practices », ECPR joint sessions, Copenhague, 14-19 avril 2000.

25. Sur la mise en forme de la politique d'information à la SDN, cf. notre ouvrage, La République contre la propagande. L'échec originel de la communication d'État en France (1918-1940), à paraître.

26. Jean de La Guérivière, Voyage à l'intérieur de l'Eurocratie, Paris, Le Monde éditions, 1992.

27. Sur ce point, cf. Gilles Bastin, «les journalistes accrédités auprès des institutions européennes à Bruxelles. Quelques changements d'un monde de travail », in Renaud Dandeu et Didier Georgakakis, L'Europe sur le métier, op.cit.

28. Gérard de Sélys, «La machine de propagande de la Commission », Le Monde diplomatique, juin 1996.

29. Cette observation est tirée d'entretiens avec des journalistes spécialistes des questions européennes.

30. Sur ces points, cf. Caroline Schickel, Les journalistes accrédités auprès de l'Union Européenne, Mémoire pour le DEA de science politique, IEP de Strasbourg, 1995.

31. Sur les processus à l'œuvre dans les secteurs journalistiques spécialisés depuis une vingtaine d'année, cf. par analogie, les travaux de Dominique Marchetti sur la santé et de Sandrine Levêque sur le social et Dominique Marchetti et Olivier Baisnée dans ce numéro.

32. Philippe Juhem, SOS Racisme. Histoire d'une mobilisation " apolitique ». Contribution à une analyse des transformations des représentations politiques après 81 , Thèse de Science Politique, Université Paris X, 1998.

33. Le Courrier international, $n^{\circ} 15,12$ au 18 novembre 1998.

34. La comparaison du traitement de la remise du rapport des sages à la veille de la démission sur la BBC et les télévisions françaises est, de ce point de vue éclairant, d'un engagement massif de la presse britannique dans la critique des institutions européennes. Sur les éditoriaux britanniques, cf., en outre, Marion Girod, Le traitement médiatique de la campagne pour les élections européennes en Grande-Bretagne, note de recherche pour le DEA de science politique sous la direction de J.-B. Legavre et D. Georgakakis, juin 1999.

35. Sur la délibération au PE cf. la thèse d'Olivier Costa, Le Parlement européen, assemblée délibérante, Paris VIII , 1998. Sur le PE comme champ périphérique, cf. Nillo Kauppi, « European Union Institution and French Political Careers ", Scandinavian Political Studies, vol.19N 11996 et la thèse en cours de Willy Beauvallet (IEP de Strasbourg).

36. Sur " points focaux » et " saillances situationnelles ", cf. T. C. Schelling, Stratégie du conflit, Paris, PUF, 1986 et M. Dobry, Sociologie des crises politiques, op.cit., p198 et suiv.

37. BQE, 5 janvier 1999.

38. Erving Goffman, Les cadres de l'expérience, Paris, Minuit, 1991.

39. Si les scandales ont moins représenté l'essor d'un espace public européen qu'un point de coordination entre différentes presses, il n'en demeure pas moins que ces mécanismes de coordination ont ouvert des possibles pour les journalistes. Pour prendre quelques exemples, le « jeu à plusieurs bandes » consistant à s'appuyer sur les révélations de « confrères " passent ici par la presse étrangère et permet de fait tous les avantages de coups indirects sans favoriser les concurrents nationaux. De même, se trouve limité l'espace des ripostes possibles des commissaires, notamment en rendant les «pressions » de face à face difficilement maîtrisables. Seuls ceux qui restent pris dans des liens nationaux exclusifs sont conduits à abandonner, comme un journaliste du Nouvel Observateur.

40. Cette paranoïa peut, au moins pour une part, être comprise comme relevant des propriétés de la situation marquée d'un côté par l'érosion d'un ensemble de soutien et de l'autre, l'illisibilité du jeu et, du même coup, l'impossibilité de voire partir les coups.

41. Sur le complot, cf. en particulier, Frédéric Monier, Le complot dans la République, Paris, La découverte, 1998. 
42. Le Conseil au sein duquel se jouent les négociations sur l'agenda 2000 et par voie de conséquence sur la réforme institutionnelle est la plus intergouvernementale des trois instances du triptyque institutionnel.

43. Pour quelques précédents, cf. M. Abélès, La vie quotidienne au Parlement européen, Paris, Hachette, 1992, p.165.

44. Successivement, tract commun des OSP, 15 novembre 1998 et BQE, 15 janvier 1999.

45. Indépendamment des unes des quotidiens européens du mois de janvier, l'examen de l'hebdomadaire de la représentation de la Commission en France, 7 jours d'Europe, en est un bon indicateur entre novembre et février.

46. Sur le recours à ces technologies en temps de crise, cf. M. Dobry, Sociologie des crises politiques, op.cit.

47. BQE, 22 janvier 1999.

48. L'API a un poids important à Bruxelles. Elle regroupe la quasi-totalité des journalistes et agit comme un instrument de communalisation du groupe. Dans l'ouvrage de Jean Nicolas, elle fait par ailleurs l'objet de constantes citations.

49. Sur ces aspects, cf. notre article, " Une mobilisation formatrice... », op.cit.

50. Le comité des sages a été désigné le 28 janvier 1999 par la conférence des présidents du Parlement européen. Il comprenait la suédoise Inga-Britt Ahlenius, auditeur général à la cour des comptes suédoise depuis 1993, l'espagnol Juan Ruanito Carrillo Salcedo, professeur de droit international, membre de la Commission européenne des Droits de l'Homme entre 1979 et 85, de la Cour européenne des Droits de l'Homme entre 1985 et 1990, le français Pierre Lelong, membre puis président de la Cour des comptes européenne entre 1977 et 89 et président de chambre à la Cour des comptes en France depuis 1994, le belge Walter van Gerven, avocat et professeur à l'Université de Louvain, et avocat à la CJCE de 1988 à 1994BQE, 29 janvier 1999.

51. BQE, 6 janvier 1999.

52. BQE, 14 janvier 1999.

53. BQE, 7 janvier 1999.

54. Marc Abélès, La vie quotidienne au Parlement européen, op.cit., p.240.

55. Paris, Presses Universitaires de France, 1968.

\section{INDEX}

Index chronologique : 1999

Mots-clés : bureaucraties/ théorie des organisations, construction européenne, crises politiques, institutions politiques et agences, sciences politiques, sociologie

Thèmes : Commission européenne

Index géographique : Bruxelles, Union européenne 